\title{
Spatial Parameterization of Infant Mortality in Anhui Province, China
}

\author{
Tonglin Zhang ${ }^{\mathrm{a}}$, Shang Xue ${ }^{\mathrm{b}}$, Ge Lin ${ }^{\mathrm{c}}$ \\ ${ }^{a}$ Department of Statistics, Purdue University, 250 North University Street, West \\ Lafayette, IN 47906, USA \\ ${ }^{b}$ Northern Trust, Corporate Risk Analytics and Insurance, Chicago, USA \\ ${ }^{c}$ Department of Environmental and Occupational Health, School of Community Health \\ Sciences, University of Nevada Las Vegas, Las Vegas, NV 89154, USA
}

\begin{abstract}
The study is motivated by an unexplained and relatively high infant mortality in Anhui Province, where a suspected unknown source may contribute to it. However, spatially focused disease clustering tests tend to based on a known location, and in many cases, use the point source as the referent. When elevated risks in some areas are caused by unknown sources, it becomes necessary to infer these sources spatially. The paper extends spatial cluster parameterization models from known sources to unknown sources by a profile likelihood method. In both simulation and extended case study, we show that the spatial parameterization method is able to effectively identify the cluster influence center and measure cluster strength. We found that the center is located where the borders of several counties met in a relatively well-to-do part of the province. In addition to its ability to identify cluster influence away from the traditional centroid point, the parameterized method tends to perform better than the common spatial cluster detection method in terms of goodness of fit statistic and location specificity.
\end{abstract}

Keywords:

Spatial parameterization, cluster point source, profile likelihood, Bootstrap

\section{Introduction}

The decline in infant mortality rates (IMRs) was the single most important factor contributing to increased life expectancy in China in the 20th 


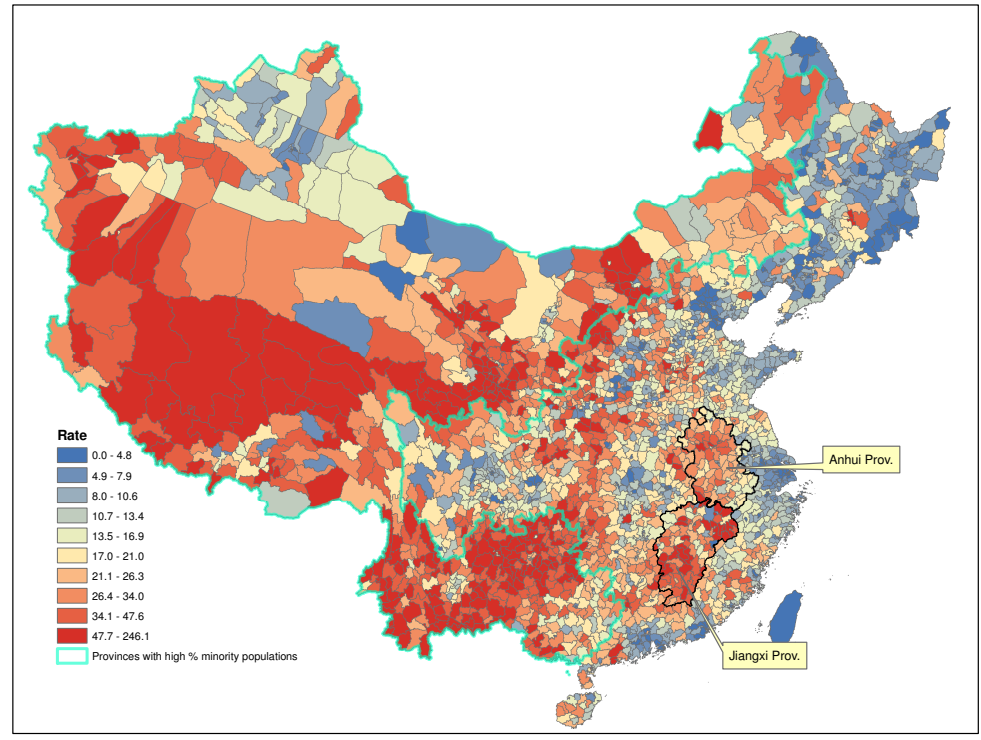

Figure 1: Infant Mortality Rate in China 2000.

century. Despite a steady decline in infant mortality over time, its geographic disparities remained. Figure 1 displays geographic variation in IMRs based on the data in 2000. It shows that some counties in western China had IMRs above 200 per 1,000, or close to the level of the early 1950s in China. It is also noted that higher IMRs are mainly around western border provinces with a relatively high proportion of minority ethnic groups. In the east, Jiangxi and Anhui Provinces have relatively high IMRs. The high IMR in Jiangxi Province has been the subject of many studies because for every one male infant death, there were more than 2 female deaths (Cai and William, 2007). In 2006, we participated in several efforts at the provincial level to detect or reconfirm spatial clusters for public health actions, especially in Jiangxi Province (Zhang, Zhang and Lin, 2012). Although Anhui Province has received less attention than Jiangxi Province, it is of interest because its IMR improved little in the 1990s and increased in relatively rich plain counties. Efforts to identify factors that cause elevated IMRs in Anhui Province started around 2000 with the United Nations Children's Funds and the Finnish government (Wu, Viisainen, and Hemminki, 2008). As part of these efforts, we assisted the Province with detecting unknown clusters and their potential influence centers. 
Several methodological issues must be considered in geographic analysis of infant mortality. First, a geographic display of infant mortality rates with substantial differences in county area may have undue influences for a county with large geographic area. In other words, an observed pattern may be spatially random even though extreme values for large areas with small populations may draw more attention (Besag and Newell, 1991). Second, multiple factors can contribute to multiple elevated risks over a large number of area units, such as provinces in China. A bottom-up approach of examining each area or region with an elevated risk may be more helpful to unpack contributing factors in each area. Third, a spatial cluster is often detected as the final deliverable, which can be achieved by both disease mapping and cluster detection methods. However, we were tasked to infer what might influence the formation of a cluster (e.g., poverty, toxic agents from air or water), and the common approach of using the centroid of an area unit as the cluster center may not be able to align exposure data spatially for the best spatial inference (Peng and Bell, 2010). It is even more problematic when 3 or 4 adjacent areas are identified as a cluster, and each contribute equally to the elevated risk. All of above issues were raised in our study of the infant mortality pattern in Anhui, China.

Since our task was to identify potential contributing sources for counties with high IMRs, and no existing method would meet our needs, we adopt a parameterized spatial cluster detection approach to infer cluster influence centers, such as point pollution sources. Most parametric cluster methods can infer cluster influence center, such as a point pollution source, but the point source is often assumed to be known (Diggle, 1990). The original method was based on a spatial point process for disease incidents and used a circle to define and test a cluster existence. The circle was extended to other shapes (Lawson, 1993), and the point process framework was later extended to generalized linear models for individual and aggregated data (Diggle and Elliott, 1997). However, this method cannot be directly applied, as we do not have the original point data. Due to time constraint at the time of the work, we used a heuristic method by partitioning the province into thousands of grid points. We then applied the parametric cluster method at each point, and identify the one with best goodness of fit statistics. Since this method is tedious, our intention in this paper is to combine the above two approaches to model cluster shape and center analytically similar to the latent spatial factor model (Wang and Wall, 2003). In the end, we can use the results from our partition method to check the consistency of our intended parameterization 
method.

To extend the cluster parameterization method from the pre-specified location to an unknown location, it is necessary to estimate an additional parameter for cluster influence point source together with cluster size and shape. Lawson (1993) noticed this problem almost two decades ago, and used a Gibbs sampler design to estimate shape, influence point, location of a potential cluster. Besides the MCMC method of Lawson, both semiparametric and non-parametric methods are able to estimate cluster size and shape parameters. For point data, a model-based kernel density method is applied to estimate the ratio of the spatial intensities of two Poisson processes (Kalsall and Diggle, 1998). A non-parametric two-dimensional scale-space smoothing method is proposed (Godtliebsen, Marron and Pizer, 2002) by extending the one-dimensional slope test-SiZer (Chauhuri and Marron, 1990). The results can be visualized as a 3D data model (e.g., contour lines, density map). Both methods have 1) a slope parameter to depict value gradient within a cluster, and 2) a bandwidth or kernel size parameter. In both cases, potential cluster sources were described or visualized, but not estimated. An insight for estimating a point source is provided by the nonparametric mode estimation method for the time-dependent models for Gaussian data (Hall and Kang, 2005), and we can consider such a problem in location estimation for Poisson data.

In this paper, we apply cluster and mode parameterization methods to estimate a potential cluster shape, size and its influence center. Our method contributes to the literature in three important ways. First, we provide a model-based point estimation method for unknown cluster locations so that geographic covariates and value variation within a cluster can be evaluated. Second, we provide a significance test for the identified cluster so that the method can be used as a parameterized cluster detection method. Third, inference can be made about a cluster influence center based on the point estimate method, so that clustered areas can be potentially linked to one or more point sources of environmental or infectious hazards (Wang and Wall, 2003). In the following sections, we first specify our modification to Diggle's original method and then evaluate it using simulated data. We then examine infant mortality with a focus on Anhui Province, and compare the results from the proposed method and Diggle's method. Finally, we offer some concluding remarks. 


\section{Methods}

\subsection{Model Specification from a Known Source to an Unknown Source}

Suppose that a study area is partitioned into $m$ area units $(i=1, \cdots, m)$, and each is associated with a number of cases $y_{i}$, the corresponding at risk population size $\xi_{i}$, and a $p$-dimensional vector of independent variables $\mathbf{x}_{i}$. Let $\lambda_{i}=E\left(y_{i}\right)$ be the expected count of the $i$-th area unit. Then, an independent Poisson model for $y_{i}$ can be written as

$$
\log \left(\lambda_{i}\right)=\log \left(\xi_{i}\right)+\mathbf{x}_{i}^{\prime} \beta
$$

where $\beta$ is a $p$-dimensional vector representing the coefficient of parameters for $\mathbf{x}_{i}$, and $\log \left(\xi_{i}\right)$ is the offset.

Model (1) does not include local variation, which can be incorporated by a spatial clustering component. For example, Gangnon and Clayton (2003) proposed a uniform spatial association term, which takes one value for areas within a cluster and another value for areas outside the cluster. When a potential spatial cluster is tested, the extent of a uniform spatial association term can be iteratively searched and added (Zhang and Lin, 2009b). A significant and positive association effect indicates a high-value cluster, and a significant and negative association indicates a low-value cluster. As the name suggests, the uniform spatial association term ignores value variation within a cluster, which can be captured by distance function from the cluster influence center (Diggle and Elliott, 1997). To ease exposition, we assume that there is a single unknown point source in the region. The idea can be easily extended if more point sources are involved. If there are more than one point, we can put a few spatial association terms in our statistical model. Each spatial association term represents a point source. We can test the significance of the point sources individually. The number of point sources can be determined by the number of significances of these point sources.

Let $d_{i}$ be the distance between the centroid of the $i$-th unit to the point source. Then, the expected count at the $i$-th area unit can be modeled by a linear function of ecological covariates and a spatial association via a loglinear model:

$$
\log \left(\lambda_{i}\right)=\log \left(\xi_{i}\right)+\mathbf{x}_{i}^{\prime} \beta+\nu h\left(d_{i}\right)
$$

where $h\left(d_{i}\right)$ describes the shape of the cluster and $\nu \geq 0$ describes the strength of a high-value cluster. This model would be Diggle and Elliott's 
model (Diggle and Elliott, 1997), if the point source were pre-specified. However, in order to uniquely define $\beta, \nu$ and $h\left(d_{i}\right)$, and therefore, the point source, we assume that $h(0)=1$ and $\lim _{d \rightarrow \infty} h(d)=0$. Following previous studies (Diggle and Rowlingson, 1994; Diggle and Elliott, 1997), we further assume that $h$ is a decreasing function on $[0, \infty)$, which can be replaced by other functions later. If there are more than one spatial cluster, then we can specify more spatial association terms in Model (2) such that each cluster can be represented by a spatial association term in the model.

The function $h$ in Model (2) can be modeled either by parametric or nonparametric methods, but our focus is on parametric functions. For a general base function $h_{0}, h$ can be modeled by $h(d)=h_{0}(d / \gamma)$, where $\gamma$ is a scalar parameter that signifies cluster influence range, and $h_{0}$ can take many distance decay forms. With the additional shape parameter $\alpha, h(d)$ may be modeled according to $h(d)=h_{0}\left[(d / \gamma)^{\alpha}\right]$. Examples include:

$$
h_{\alpha, \gamma}(d)=e^{-(d / \gamma)^{\alpha}}, \alpha, \gamma>0
$$

for the exponential decay function $h_{0}(d)=e^{-d}$,

$$
h_{\alpha, \gamma}(d)=\left[1+(d / \gamma)^{\alpha}\right]^{-1}, \alpha, \gamma>0
$$

for the polynomial decay function $h_{0}=(1+d)^{-1}$, and

$$
h_{\alpha, \gamma}(d)=[1+\alpha \log (d / \gamma)]^{-1}, \alpha, \gamma>0
$$

for the logarithm decay function $h_{0}(d)=[1+\log (d)]^{-1}$. The distance $d$ can be either modeled isotropically or anisotropically. When an isotropic distance is used, the cluster defined in Model (2) is circular. When an anisotropic distance is used, the cluster could be in other forms, such as ellipse or asymmetric shapes.

Even though the above base functions are unbounded, a bounded function can be considered by taking a base function $h_{0}$ that satisfies $h_{0}(d)=0$ if $d \geq 1$ and $h_{0}(d)>0$ if $d<1$. Examples could be

$$
h_{\alpha, \gamma}(d)=\left[1-(d / \gamma)^{\alpha}\right] I_{[0,1]}(d / \gamma)
$$

for the base function $h_{0}(d)=(1-d) I_{[0,1]}(d)$, where $I_{[0,1]}$ is the indicator function. When $\alpha=0$, we have a special case for the uniform association term (Gangnon and Clayton, 2003). Since a "cluster" can be presented by a 


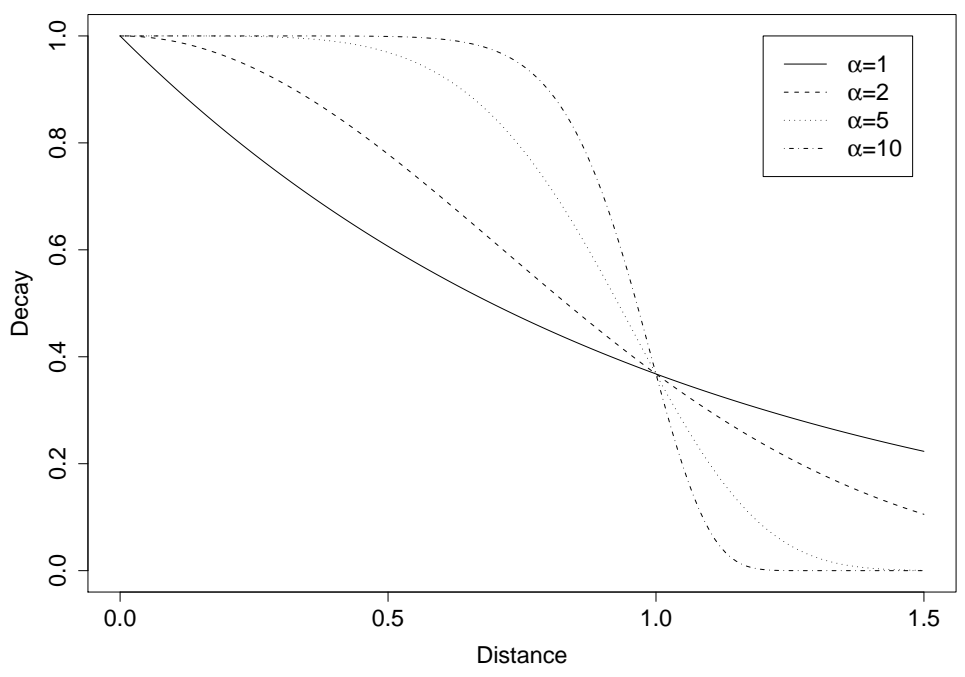

Figure 2: Exponential Decay Functions.

bounded or unbounded function, we use it more generically without distinguishing between the two in this section, and point out their differences in the application study when it becomes necessary.

We use the exponential decay function in (3) to demonstrate our approach with most parameters being described before. When $h$ is parameterized by the $h_{\alpha, \gamma}$ in Model (3), Model (2) becomes

$$
\log \left(\lambda_{i}\right)=\log \left(\xi_{i}\right)+\mathbf{x}_{i}^{\prime} \beta+\nu e^{-\left(d_{i} / \gamma\right)^{\alpha}} .
$$

Figure 2 displays distance decay curves for selected $\alpha$ values that depict cluster strength exponentially. First, let's consider extreme $\alpha$ values not shown in the Figure. When the shape parameter $\alpha$ goes to 0 , values within a cluster decline rapidly in a spikey shape. On the other extreme, a flat shape is applied when $\alpha$ goes to $\infty$, and its effect is almost identical to the uniform association. In between the two, $\alpha$ values describe the steepness of the decrease either closer or farther from the center, as shown in cases of $\alpha=$ $1,2,5,10$. As in model-based geostatistics, the shape parameter $\alpha$ is assumed unknown, and its parameter estimate captures unobserved risk factors that affect the cluster shape similar to different bandwidths in geostatistics.

Let $\mathbf{a}_{i}=\left(a_{i 1}, a_{i 2}\right) \in \mathbb{R}^{2}$ be the centroid of the $i$-th unit and $\mathbf{a}_{0}=$ $\left(a_{01}, a_{02}\right) \in \mathbb{R}^{2}$ be the point source. When it is unknown, $\mathbf{a}_{0}$ is a mode 
parameter (or vector). Its variation captures the trend surface of the relative risk. In addition, when the third term in Model (2) is parameterized by $h_{0}\left(d_{i} / \gamma\right)$ for a known base function $h_{0}$, it needs to include a scale (or range) parameter $\gamma$ and a strength parameter $\nu$.

The scale parameter $\gamma$ describes the size of the cluster. If the size of the cluster is large, then $\gamma$ is also large. The relative risk at a point in the study area decreases as the distance between the point to the cluster center increases. The value of the relative risk is $\nu h(\gamma)=\nu h_{0}(1)$ if the distance is $\gamma$ and declines to $\nu h_{0}(2)$ when the distance becomes $2 \gamma$. If $h_{0}$ is specified as an exponential decay function as in Model (5), their values are $0.2678 \nu$ and $0.1353 \nu$ respectively.

The strength parameter $\nu$ determines cluster value elevation. Its sign determines whether the cluster is a hotspot $(\nu>0)$ or a cool spot $(\nu<0)$. As $|\nu|$ increases, the value strengthens. As $\nu \rightarrow 0$, the cluster flattens toward a weak cluster. When $\nu=0$, Model (2) reduces to (1), which indicates nonexistence of spatial clusters.

Note also that once $\alpha>0, \gamma>0$ and $\mathbf{a}_{0}$ are specified as unknown parameters, Model (2) is no longer a loglinear model (Agresti, 1990), because the third term in the model depends on parameters $\gamma$ and $\mathbf{a}_{0}$. However, when $\mathbf{a}_{0}, \gamma$ and $\alpha$ are known, Model (2) becomes a generalized linear model defined by a linear function of $\beta$ and $\nu$ only. Therefore, Model (2) contains both linear and nonlinear components of unknown parameters: if parameters related to $h\left(d_{i}\right)$ are all known, Model (2) is a generalized linear model; otherwise, it is a generalized nonlinear model.

\subsection{Parameter estimation}

The estimation process has to consider both the liner and nonlinear components. Assume $y_{1}, \cdots, y_{m}$ are independent Poisson random variables. For a given $h=h_{\alpha, \nu}$, the loglikelihood function of Model (2) is:

$$
\begin{aligned}
\ell\left(\beta, \nu, \gamma, \mathbf{a}_{0}, \alpha\right)= & -\sum_{i=1}^{m} \log \left(y_{i} !\right)+\sum_{i=1}^{m} y_{i}\left[\log \left(\xi_{i}\right)+\log \left(\mathbf{x}_{i}^{\prime} \beta\right)+\nu h_{\alpha, \gamma}\left(d_{i}\right)\right] \\
& -\sum_{i=1}^{m} \exp \left[\log \left(\xi_{i}\right)+\mathbf{x}_{i}^{\prime} \beta+\nu h_{\alpha, \gamma}\left(d_{i}\right)\right] .
\end{aligned}
$$

By maximizing Equation (6), we derived the maximum likelihood estimators of parameters. To easily express the method, we denote $\eta=\left(\beta, \nu, \gamma, \mathbf{a}_{0}, \alpha\right)=$ 
$(\theta, \alpha)$ and write the loglikelihood function in Equation (6) as $\ell(\eta)$. Then, the MLE of $\eta$ can be solved by $\dot{\ell}(\eta)=0$. With an initial guess $\ell^{(0)}$ for $\eta$, the MLE may be derived by a Newton-Raphson algorithm as

$$
\eta^{(k+1)}=\eta^{(k)}-\ddot{\ell}^{-1}\left(\eta^{(k)}\right) \dot{\ell}\left(\eta^{(k)}\right),
$$

where $\ell^{(k)}$ is the value of $\eta$ in the $k$ th step in the algorithm, $\dot{\ell}(\eta)$ is the gradient and $\ddot{\ell}(\eta)$ is the Hessian matrix of $\ell(\eta)$.

In order to understand the performance of our method, we evaluate the asymptotic property of $\hat{\eta}$ when $\lambda_{\min }=\min \left(\lambda_{i}\right)$ is large. We find that the parameters $\gamma, \mathbf{a}_{0}$, and $\alpha$ are only present when $\nu>0$, which implies that the asymptotic distribution of $\hat{\eta}$ is irregular if $\nu=0$ (Davies, 1977). Therefore, we decided to evaluate the performance of $\hat{\eta}$ under the case when $\nu>0$. To simplify our notation, we write $\eta=(\theta, \alpha)=\left(\beta, \nu, \gamma, \mathbf{a}_{0}, \alpha\right)$ and $\hat{\eta}$ as the MLE of $\eta$. Then, $\hat{\eta}$ is derived by maximizing Equation (6) for $\eta$. To derive the asymptotic normality for $\eta$, we need the following regularity condition:

\section{Regularity Conditions:}

(C1) The domain of $\eta$ is an open subset of $\mathbb{R}^{p+5}$ and $\nu_{0}>0$, where $\eta_{0}=$ $\left(\beta_{0}, \nu_{0}, \gamma_{0}, \mathbf{a}_{00}, \alpha_{0}\right)$ is the true vector of $\eta$ (i.e., $\beta_{0}, \nu_{0}, \gamma_{0}, \mathbf{a}_{00}$ and $\alpha_{0}$ are true values of $\beta, \nu, \gamma, \mathbf{a}_{0}$, and $\alpha$, respectively).

(C2) The second-oder partial derivatives of $\ell(\eta)$ are continuous and their expected values always exist.

(C3) The Fisher Information Matrix $I_{0}(\eta)=I(\eta) / \lambda_{\min }=-E[\ddot{\ell}(\eta)] / \lambda_{\min }$ is positive definite within a neighborhood of $\eta_{0}$

(C4) Model (2) (with $h\left(d_{i}\right)=h_{\alpha, \gamma}\left(d_{i}\right)$ ) satisfies the uniqueness condition: we always have $\beta=\beta^{\prime}, \gamma=\gamma^{\prime}, \nu=\nu^{\prime}, \alpha=\alpha^{\prime}$, and $\mathbf{a}_{0}=\mathbf{a}_{0}^{\prime}$ if $\mathbf{x}_{i} \beta+\nu h_{\alpha, \gamma}\left(d_{i}\right)=\mathbf{x}_{i} \beta^{\prime}+\nu^{\prime} h_{\alpha^{\prime}, \gamma^{\prime}} h\left(d_{i}^{\prime}\right)$ for any $i=1, \cdots, m$, where $d_{i}=\left\|\mathbf{a}_{i}-\mathbf{a}_{0}\right\|$ and $d_{i}^{\prime}=\left\|\mathbf{a}_{i}-\mathbf{a}_{0}^{\prime}\right\|$.

The above regularity conditions are modified from the usual regularity conditions of the asymptotic normality of iid sample (e.g. Rao (1973), P 364; Ferguson (1996), P 121).

Theorem 1. If Conditions (C1)-(C4) hold, then $\hat{\eta}$ is consistent and

$$
\lambda_{\text {min }}^{\frac{1}{2}}\left(\hat{\eta}-\eta_{0}\right) \stackrel{D}{\rightarrow} N\left(0, I_{0}^{-1}\left(\eta_{0}\right)\right)
$$

as $\lambda_{\min } \rightarrow \infty$. 
Proof: Denote $\kappa=\left[\lambda_{\text {min }}\right]$ as the integer part of $\lambda_{\text {min }}$. We equivalently rephrase the case when $\lambda_{\text {min }} \rightarrow \infty$ by partitioning $y_{i}=\sum_{i=1}^{\kappa} y_{i k}$, where $Y_{i k}$ are iid Poisson random variables with common mean $\lambda_{i} / \kappa$. For a given $k$, the loglikelihood of $\eta$ based on $\left(y_{1 k}, \cdots, y_{m k}\right)$ is

$$
\begin{aligned}
\ell_{k}(\eta)= & -\sum_{i=1}^{m} \log \left(y_{i k} !\right)+\sum_{i=1}^{m} y_{i k}\left[\log \left(\xi_{i}\right)-\log (\kappa)+\log \left(\mathbf{x}_{i}^{\prime} \beta\right)+\nu h_{\alpha, \gamma}\left(d_{i}\right)\right] \\
& -\frac{1}{\kappa} \sum_{i=1}^{m} \exp \left[\log \left(\xi_{i}\right)+\mathbf{x}_{i}^{\prime} \beta+\nu h_{\alpha, \gamma}\left(d_{i}\right)\right] .
\end{aligned}
$$

For each fixed $\eta, \ell_{k}(\eta), k=1, \cdots, \kappa$, are iid random variables with comment expected value $\varphi(\eta)=E\left[\ell_{k}(\eta)\right]$. By the Jensen Inequality, there is $\varphi(\eta) \leq$ $\varphi\left(\eta_{0}\right)$ and by Condition (C4) the equality holds if and only if $\eta=\eta_{0}$. The loglikelihood function of $\left(y_{1 k}, \cdots, y_{m k}\right)$ for all $k=1, \cdots, \kappa$ is

$$
\begin{aligned}
\tilde{\ell}(\eta)= & \sum_{k=1}^{\kappa} \ell_{k}(\eta) \\
= & -\sum_{k=1}^{\kappa} \sum_{i=1}^{m} \log \left(y_{i k} !\right)+\sum_{k=1}^{\kappa} \sum_{i=1}^{m} y_{i k}\left[\log \left(\xi_{i}\right)-\log (\kappa)+\log \left(\mathbf{x}_{i}^{\prime} \beta\right)+\nu h_{\alpha, \gamma}\left(d_{i}\right)\right] \\
& -\sum_{i=1}^{m} \exp \left[\log \left(\xi_{i}\right)+\mathbf{x}_{i}^{\prime} \beta+\nu h_{\alpha, \gamma}\left(d_{i}\right)\right. \\
= & \ell(\eta)+\sum_{i=1}^{m} \log \left(y_{i} !\right)-\sum_{k=1}^{\kappa} \sum_{i=1}^{m} \log \left(y_{i k} !\right) .
\end{aligned}
$$

By the strong law of large number (SLLN) and the Shannon-Kolmogorov Information Inequality (also known as the nonnegativity of the KullbackLeibler divergence (Ferguson (1996), P 113), we have

$$
\frac{1}{\kappa}\left[\ell(\eta)-\ell\left(\eta_{0}\right)\right]=\frac{1}{\kappa}\left[\tilde{\ell}(\eta)-\tilde{\ell}\left(\eta_{0}\right)\right] \stackrel{P}{\rightarrow} \varphi(\eta)-\varphi\left(\eta_{0}\right) \leq 0
$$

and the equality hold if and only if $\eta=\eta_{0}$. Therefore, $\hat{\eta}$ is consistent. Now, we consider the performance of $\dot{\ell}_{k}\left(\eta_{0}\right)$. Then, $E\left[\dot{\ell}_{k}\left(\eta_{0}\right)\right]=0$ and $V\left[\dot{\ell}_{k}\left(\eta_{0}\right)\right]=$ $I\left(\eta_{0}\right)$ (Ferguson (1996), P 120). Since $\dot{\ell}_{k}\left(\eta_{0}\right)$ for $k=1, \cdots, \kappa$ are iid, we have $\kappa^{-\frac{1}{2}} \dot{\ell}\left(\eta_{0}\right) \stackrel{D}{\rightarrow} N\left(0, I_{0}\left(\eta_{0}\right)\right)$. To derive (8), we consider the Taylor expansion of 
$\dot{\ell}\left(\eta_{0}\right)$ at $\hat{\eta}$ and obtain

$$
\begin{aligned}
\dot{\ell}\left(\eta_{0}\right) & =\dot{\ell}(\hat{\eta})+\ddot{\ell}(\hat{\eta})\left(\eta_{0}-\hat{\eta}\right)+o_{p}\left(\left\|\eta_{0}-\hat{\eta}\right\|\right) \\
& =\ddot{\ell}\left(\eta_{0}\right)\left(\eta_{0}-\hat{\eta}\right)+o_{p}\left(\left\|\eta_{0}-\hat{\eta}\right\|\right) .
\end{aligned}
$$

Then,

$$
\sqrt{\kappa}\left(\hat{\eta}-\eta_{0}\right)=\left[\kappa \ddot{\ell}\left(\eta_{0}\right)\right]^{-1} \kappa^{-\frac{1}{2}} \dot{\ell}\left(\eta_{0}\right)+o_{p}(1)
$$

and Equation (8) is derived since we have $\kappa^{-1} \ddot{\ell}\left(\eta_{0}\right) \stackrel{P}{\rightarrow} I_{0}\left(\eta_{0}\right)$ by SLLN and $\kappa / \lambda_{\text {min }} \rightarrow 1$ as $\lambda_{\text {min }} \rightarrow \infty$ by the definition of $\kappa$.

Since the dimension of $\eta$ is often high in applications, the algorithm given by Equation (7) is hard to be applied. Thus, we propose another algorthm. We note that Equation (6) can be directly maximized by the usual GLM fitting procedure Faraway (2005) if parameters in $h_{\alpha, \gamma}\left(d_{i}\right)$ are completely known. Therefore, we focus on the development of algorithm for parameters in the nonlinear component. Since the estimation of the shape parameter $\alpha$ significantly influences the stability of the estimation of other parameters, we first optimizes the likelihood function for a known $\alpha$ by assuming that $\alpha$ is given, and then optimizes the likelihood function for $\alpha$ by relaxing this assumption.

Let the unknown parameters be $\theta=\left(\beta, \nu, \gamma, \mathbf{a}_{0}\right)=\left(\theta_{1}, \theta_{2}\right)$, where $\theta_{1}=$ $(\beta, \nu)$ and $\theta_{2}=\left(\gamma, \mathbf{a}_{0}\right)$. When $\theta_{2}$ is given, Model (5) is a usual generalized linear model. Based on this property, we can compute the full MLE by the following algorithm:

\section{The algorithm of the fitting procedure:}

i) Compute the MLE of $\theta_{1}^{(0)}$ by assuming $\nu=0$. Then, we derived $\theta^{(0)}=$ $\theta_{1}^{(0)}$ because in this case $\theta_{2}$ is not considered.

ii) Assume $\theta^{(k-1)}$ is derived at the $k$-th step. Compute the conditional MLE of $\theta_{2}$ given $\theta_{1}^{(k-1)}$. Let it be $\theta_{2}^{(k)}$. Compute the conditional MLE of $\theta_{1}$ given $\theta_{2}^{(k)}$. Let it be $\theta_{1}^{(k)}$. Let $\theta^{(k)}=\left(\theta_{1}^{(k)}, \theta_{2}^{(k)}\right)$.

iii) Iterate step ii) until convergence.

This estimation process is analogous to the usual profile likelihood fitting procedure for the full MLE that "takes the supremum in two steps" (Murphy and van der Vaart, 2000). To reduce dimension of the likelihood function, our method also takes the supremum in two steps. Unlike the profile likelihood 
method, which only computes the conditional maximization, our method computes the full MLE based on the two conditional maximizations. When the MLE is derived, the variance-covariance matrix can be derived by using the estimate of the Fisher Information matrix.

Since the conditional MLE of $\theta_{1}$ given $\theta_{2}$ can be directly estimated by the standard GLM fitting procedure, we concentrate on only the computation of the conditional MLE of $\theta_{2}$ given $\theta_{1}$. Let $\dot{\ell}_{\theta_{2}}\left(\theta_{1}, \theta_{2}, \alpha\right)=\dot{\ell}_{\theta_{2}}(\eta)=\partial \ell(\eta) / \partial \theta_{2}$ be the vector of the first order partial derivatives of the loglikelihood function $\ell$ with respect to $\theta_{2}$ and $\ddot{\ell}_{\theta_{2}}\left(\theta_{1}, \theta_{2}, \alpha\right)=\ddot{\ell}_{\theta_{2}}(\eta)=\partial^{2} \ell(\eta) / \partial \theta_{2}^{2}$ be the corresponding Hessian matrix. Then, the conditional MLE of $\theta_{2}$ given $\theta_{1}$ can be iteratively derived by the Newton-Raphson method as

$$
\theta_{2}^{(v+1)}=\theta_{2}^{(v)}-\ddot{\ell}_{\theta_{2}}^{-1}\left(\theta_{1}^{(v-1)}, \theta_{2}^{(v-1)}, \alpha\right) \dot{\ell}_{\theta_{2}}\left(\theta_{1}^{(v-1)}, \theta_{2}^{(v-1)}, \alpha\right),
$$

where $\theta_{2}^{(v)}$ is the $k$-th iterative solution to equation $\dot{\ell}_{\theta_{2}}\left(\theta_{1}^{(v-1)}, \theta_{2}, \alpha\right)=0$ for given $\theta_{1}^{(v-1)}$ and $\alpha$. Analytically, the partial derivative of $\theta_{2}$ can be derived as follows:

$$
\begin{aligned}
\frac{\partial \ell}{\partial \gamma} & =\sum_{i=1}^{m}\left[y_{i} \nu-\nu e^{\log \left(\xi_{i}\right)+\mathbf{x}_{i}^{\prime} \beta+\nu h_{\gamma}\left(d_{i}\right)}\right] \frac{\partial h_{\gamma}\left(d_{i}\right)}{\partial \gamma} \\
\frac{\partial \ell}{\partial a_{0 j}} & =\sum_{i=1}^{m}\left[y_{i} \nu-\nu e^{\log \left(\xi_{i}\right)+\mathbf{x}_{i}^{\prime} \beta+\nu h_{\gamma}\left(d_{i}\right)}\right] \frac{\partial h_{\gamma}\left(d_{i}\right)}{\partial d_{i}} \frac{\partial d_{i}}{\partial a_{0 j}}, j=1,2 .
\end{aligned}
$$

Based on the analytical procedure above, the second order partial derivatives can be derived accordingly.

To apply the above algorithm, it is important to have an ideal initial guess $\theta_{2}^{(0)} \in \mathbb{R}^{2} \times \mathbb{R}^{+}$. Here we consider a global searching procedure which is modified from the three-dimensional golden section algorithm.

The global searching procedure for the initial guess $\theta_{2}^{(0)}$ :

i) Suppose the best $\theta_{2}=\left(a_{01}, a_{02}, \gamma\right)$ is within $\left[a_{011}, a_{012}\right] \times\left[a_{021}, a_{022}\right] \times$ $\left[\gamma_{1}, \gamma_{2}\right]$

ii) At the $r$ th step, compute the value of $\ell(\eta)$ for $a_{01}=a_{011}^{(r-1)}+i\left(a_{012}^{(r+1)}-\right.$ $\left.a_{011}^{(r-1)}\right) / 4, a_{02}=a_{011}^{(r-1)}+j\left(a_{012}^{(r-1)}-a_{011}^{(r-1)}\right) / 4$, and $\gamma=\gamma_{1}^{(r-1)}+k\left(\gamma_{2}^{(r-1)}-\right.$ $\left.\gamma_{1}^{(r-1)}\right) / 4$, where $i, j, k=0,1,2,3,4$.

iii) Suppose $\ell(\eta)$ is maximized at $\left(i_{0}, j_{0}, k_{0}\right)$. If $i_{0}=0,1$, then update $a_{011}^{(r)}=a_{011}^{(r-1)}$ and $a_{012}^{(r)}=a_{011}^{(r-1)}+\left(a_{012}^{(r-1)}-a_{012}^{(r-1)}\right) / 2$; if $r=2$, then update 
$a_{011}^{(r)}=a_{011}^{(r-1)}+\left(a_{012}^{(r-1)}-a_{012}^{(r-1)}\right) / 4$ and $a_{012}^{(r)}=a_{011}^{(r-1)}+3\left(a_{012}^{(r-1)}-a_{012}^{(r-1)}\right) / 4$; if $r=3,4$, then update $a_{011}^{(r)}=a_{011}^{(r-1)}+\left(a_{012}^{(r-1)}-a_{012}^{(r-1)}\right) / 2$ and $a_{012}^{(r)}=$ $a_{012}^{(r-1)}$. Update $a_{021}^{(r)}, a_{022}^{(r)}$ and $\gamma_{1}^{(r)}, \gamma_{2}^{(r)}$ using the similar way.

iv) Repeat Steps ii) and iii) a few more times for the initial guess $\theta_{2}^{(0)}$.

In the searching procedure for $\theta_{2}^{(0)}$, the side length of the region is reduced by half at each step. A good choice of $\theta_{2}^{(0)}$ can be identified if the region is small enough. After that, we use the Newtow-Raphson algorithm since its convergence rate is fast. Because the consideration for $\theta_{2}^{(0)}$ is based on the global maximization criterion, the final answer for $\theta_{2}$ in the Newton-Raphson algorithm should be the global maximum.

For a given $\alpha$, as long as $\hat{\theta}$ is derived, we can use Equation (8) to compute the confidence bound for $\theta$, where we approximately use

$$
\hat{\theta}-\theta_{0} \sim^{\text {approx }} N\left(0, I\left(\hat{\theta}: \hat{\eta}_{\alpha}\right)\right),
$$

where $\hat{\eta}_{\alpha}$ is the MLE of $\eta$ given $\alpha$ and $I(\theta: \eta)$ is sub-matrix of $I(\eta)$ for $\theta$.

Because the centroid parameter $\mathbf{a}_{0}$ is more important than the rest parameters, we provide a way to compute the confidence bound for $\mathbf{a}_{0}$ which is given by

$$
C\left(\mathbf{a}_{0}\right)=\left\{\mathbf{a}_{0}:\left(\hat{\mathbf{a}}_{0}-\mathbf{a}_{0}\right)^{\prime} I^{-1}\left(\hat{\mathbf{a}}_{0} ; \hat{\eta}_{\alpha}\right)\left(\hat{\mathbf{a}}_{0}-\mathbf{a}_{0}\right) \leq \chi_{\alpha, 2}^{2}\right\},
$$

where $\chi_{\alpha, 2}^{2}$ is the upper $\alpha$-quantile of the $\chi_{2}^{2}$ distribution, $\hat{\eta}_{\alpha}=(\hat{\theta}, \alpha)$ is the MLE of $\eta$ for a given $\alpha$, and $I\left(\mathbf{a}_{0}: \eta\right)$ is the sub-matrix of $I(\eta)$ for $\mathbf{a}_{0}$.

Finally, we relax the shape parameter $\alpha$ to be unknown, and compute the best $\alpha$ according to the Pearson and deviance goodness of fit statistics. We assume that the best $\alpha$ minimizes the value of $G_{\alpha}^{2}$ or $X_{\alpha}^{2}$ respectively, where $G_{\alpha}^{2}$ and $X_{\alpha}^{2}$ are residual deviance or Pearson goodness-of-fit statistics, respectively. We use the golden section algorithm to search the best $\alpha$. The algorithm is given below:

The golden section algorithm for the best $\alpha$ based on $G_{\alpha}^{2}$ :

i) Suppose the best $\alpha$ is between $\alpha_{1}^{(0)}$ and $\alpha_{2}^{(0)}$ with $\alpha_{1}^{(0)}<\alpha_{2}^{(0)}$.

ii) At the $k$-th step, compute the value of $G_{\alpha}^{2}$ for $\alpha=\alpha_{1}^{(k-1)}+i\left(\alpha_{2}^{(k-1)}-\right.$ $\left.\alpha_{1}^{(k-1)}\right) / 4$ for $i=0,1,2,3,4$. 
iii) If $G_{\alpha}^{2}$ has its minimum value at $i=0$ or 1 , then update $\alpha_{1}^{(k)}=\alpha_{1}^{(k-1)}$ and $\alpha_{2}^{(k)}=\alpha_{1}^{(k-1)}+\left(\alpha_{2}^{(k-1)}-\alpha_{1}^{(k-1)}\right) / 2$; if $G_{\alpha}^{2}$ has its minimum value at $i=2$, then update $\alpha_{1}^{(k)}=\alpha_{1}^{(k-1)}+\left(\alpha_{2}^{(k-1)}-\alpha_{1}^{(k-1)}\right) / 4$ and $\alpha_{2}^{(k)}=$ $\alpha_{1}^{(k-1)}+3\left(\alpha_{2}^{(k-1)}-\alpha_{1}^{(k-1)}\right) / 4$; if $G_{\alpha}^{2}$ has its minimum value at $i=3$ or 4 , then update $\alpha_{1}^{(k)}=\alpha_{1}^{(k-1)}+\left(\alpha_{2}^{(k-1)}-\alpha_{1}^{(k-1)}\right) / 2$ and $\alpha_{2}^{(k)}=\alpha_{2}^{(k-1)}$.

iv) Iterate Steps ii) and iii) until convergence.

The algorithm is able to find the best $\alpha$ if $G_{\alpha}^{2}$ has a unique local minimum between $\alpha_{1}^{(0)}$ and $\alpha_{2}^{(0)}$. The algorithm form $X_{\alpha}^{2}$ can be proposed similarly. The method based on $G_{\alpha}^{2}$ coincides with the likelihood-based approach. The method based on $X_{\alpha}^{2}$ is considered as an alternative approach. In practice, we can simply choose a very small value for $\alpha_{0}^{(0)}$ and a very large value for $\alpha_{2}^{(0)}$ in the algorithm.

In summary, the fitting procedure yields consistent estimator of unknown parameters for Equation (6) when the model is correctly specified for the base function $h_{0}$ and the linear function $\mathbf{x}_{i}^{\prime} \beta$. If the specification of $h_{0}$ is unknown, one can specify a number of candidates from the base functions $h_{0}$ and select the one with the best goodness of fit statistic, such as the minimum value of the Pearson $\chi^{2}$ statistic or the loglikelihood ratio statistic. A natural way is to compare the values of $X_{\hat{\alpha}}^{2}$ or $G_{\hat{\alpha}}^{2}$ for different choices of $h_{0}$. The best choice of $h_{0}$ is identified by minimizing the $X_{\hat{\alpha}}^{2}$ or $G_{\hat{\alpha}}^{2}$ value.

\subsection{Cluster detection}

The theorem and procedure provided in Section 2.2 focus on the case when $\nu>0$, which is regular. In classical cluster detection problems, such as the spatial scan statistic problem, the fundamental question is to answer whether $\nu=0$ or not, which cannot be answered by the theorem and procedure provided in Section 2.2 because these problems are irregular (Davies, 1977). For this reason, we consider the classical cluster detection problem in this section, where the fitting procedure is useful in the computation of the likelihood ratio statistic.

The significance of a cluster can be evaluated by testing the null hypothesis $H_{0}: \nu=0$ against the alternative hypothesis $H_{1}: \nu>0$. We use the likelihood ratio statistic given by

$$
\Lambda=\frac{\sup _{\nu \geq 0} L(\eta)}{\sup _{\nu=0} L(\eta)}
$$


where $\sup _{\nu \geq 0} L(\eta)$ or $\sup _{\nu=0} L(\eta)$ is the supremum of the likelihood function $L(\eta)$ under the union of the null and the alternative hypotheses or under the null hypothesis respectively. Both the numerator and the denominator of Equation (11) can be derived by

$$
\sup _{\nu \geq 0} L(\eta)=L(\hat{\eta})
$$

and

$$
\sup _{\nu=0} L(\eta)=L\left(\hat{\eta}_{\nu=0}\right)
$$

where $\hat{\eta}$ is the MLE of $\eta$ under Model (2) and $\hat{\eta}_{\nu=0}$ is the MLE of $\eta$ under Model (1). The algorithm to compute the likelihood ratio statistic $\Lambda$ is straightforward. When $\nu=0$, Model (2) reduces to Model (1), or a usual loglinear model. In this case $\hat{\eta}_{\nu=0}$ becomes $\hat{\beta}_{\nu=0}$ and can be derived from the usual loglinear model. When $\nu \geq 0$, the method to compute $\hat{\eta}$ in Section 2.2 can be use instead. The null hypothesis of $H_{0}: \nu=0$ is rejected if the value of $\Lambda$ is large. Equation (11) can also be used for a given $\alpha$, because for a given $\alpha$ it becomes

$$
\Lambda=\frac{\sup _{\nu \geq 0 \mid \alpha} L(\eta)}{\sup _{\nu=0 \mid \alpha} L(\eta)}=\frac{L\left(\hat{\theta}_{\alpha}, \alpha\right)}{L\left(\hat{\eta}_{\nu=0}\right)}
$$

where $\hat{\theta}_{\alpha}$ is the conditional MLE of $\theta$ given $\alpha$ and $\hat{\eta}_{\nu=0}$ only contains the MLE of $\beta$ under $\nu=0$. Since $\sup _{\nu=0} L(\eta)=\sup _{\nu>0, \gamma=0} L(\eta)$, the case $\nu>0$ with $\gamma=0$ is also included in the null hypothesis.

The computation of the $p$-value of $\Lambda$ requires the full specification of its exact or asymptotic distribution under the null hypothesis. Since the parameters $\gamma$ and $\mathbf{a}_{0}$ are absent when $\nu=0$, the likelihood ratio statistic $\Lambda$ does not process its usual $\chi^{2}$ asymptotic distribution. We, therefore, assess the $p$-value of $\Lambda$ based on the bootstrap method of spatial scan statistic (Boos, 2003; Kulldorff, 1997).

The bootstrap method:

(a) Fit Model (1) and derive the estimate of $\hat{\beta}_{H_{0}}$ and compute $\Lambda$ using Equation (11).

(b) Generate $K$ datasets from Model (1) with parameter $\hat{\beta}_{H_{0}}$ by assuming $y_{i} \sim \operatorname{Poisson}\left(\xi_{i} e^{\mathbf{x}_{i}^{\prime} \hat{\beta}_{H_{0}}}\right)$ independently and compute the value of $\Lambda_{k}^{*}$ in each generated dataset. 
(c) The $p$-value of $\Lambda$ is given by its rank in $\left\{\Lambda_{k}^{*}: k=0, \cdots, K\right\}$, where $\Lambda_{0}^{*}=\Lambda$.

\section{Simulation}

In this section, we evaluated the power and location specificity of the proposed method. We partitioned a unit square in $\mathbb{R}^{2}$ into one hundred 0.1 unit grids and randomly generated a point within each grid. This design mimics the real-world situation, in which the centroid of an area unit such as a county may not necessarily be the center of a cluster. In addition, we fixed the cluster influence center at $\mathbf{a}_{0}=(0.5,0.5)$ and wrote $a_{01}=a_{02}=0.5$. This design mimics the influence of a hypothetical point source (e.g., point of toxic release) at a location that is not necessarily co-centered with a grid center. Consequently, each point on a grid had its distance to the center

$$
d_{i}=\left\|\mathbf{a}_{i}-\mathbf{a}_{0}\right\|=\sqrt{\left(a_{i 1}-0.5\right)^{2}+\left(a_{i 2}-0.5\right)^{2}}
$$

where $\mathbf{a}_{i}=\left(a_{i 1}, a_{i 2}\right)$ represents the 100 points that were generated from the 100 grids.

In order to have a reasonable population size, we iid generated population $\left(\xi_{1}, \cdots, \xi_{100}\right)$ for each unit from the nearest integer of $100+\operatorname{Gamma}(10,0.001)$, so that $\xi_{i} \geq 100, E\left(\xi_{i}\right)=10100$ and $V\left(\xi_{i}\right)=10^{7}$. In order to have case counts distributed in a decreasing function according to their distances to $\mathbf{a}_{0}$, we independently generated Poisson random counts $y_{1}, \cdots, y_{100}$ conditional on $\xi_{1}, \cdots, \xi_{100}$, with the expected values $\lambda_{1}, \cdots, \lambda_{100}$ being

$$
\log \left(\lambda_{i}\right)=\log \left(\xi_{i}\right)+\beta_{0}+\nu e^{-\left(d_{i} / \gamma\right)^{2}} .
$$

We fixed $\beta_{0}=-7$ and $\gamma=0.2$, so that the expected count for a location far away from $a_{0}$ (or for a cluster strength $\nu=0$ ) is

$$
E\left(y_{i}\right)=E\left[E\left(y_{i} \mid \xi_{i}\right)\right]=E\left(e^{-7} \xi_{i}\right)=e^{-7} \times 10100=9.21,
$$

and its variance is

$$
V\left(y_{i}\right)=E\left[V\left(y_{i} \mid \xi_{i}\right)\right]+V\left[E\left(y_{i} \mid \xi_{i}\right)\right]=E\left(e^{-7} \xi_{i}\right)+V\left(e^{-7} \xi_{i}\right)=17.53
$$

The expected value and variance were chosen as such to avoid too many zeros or many large counts in the simulated data. We chose $\gamma=0.2$ because the relative risk at the center of the cluster was over 10 times $\left(e^{0.5 / 0.2}=12.18\right)$ 
greater than the relative risk at the boundary of the unit square. Therefore, the boundary points in the unit square could be thought as outside of the cluster.

We generated 1,000 sets of Poisson counts $y_{1}, \cdots, y_{100}$ for each $\nu$ value from 0 to 2 in step 0.1 , in which $\nu=0$ corresponds to the null hypothesis of no spatial cluster, and $\nu=2$ corresponds to a strong cluster pulling power for an elevated risk. As the estimate of $\alpha$ was time-consuming, we treated $\alpha$ as a known parameter. We fixed the distance decay parameter $\alpha$ at 2 based on findings from the previous literature (Diggle and Elliott, 1997), so that we were able to estimate $\nu, \beta_{0}, \gamma, a_{01}$ and $a_{02}$. We had also investigated the algorithm for estimation of $\alpha$ given by the previous section and found that most estimates of $\alpha$ were between 1.5 and 2.5. Since we found that the impact of $\alpha$ on goodness of fit statistics (i.e. $X_{\alpha}^{2}$ or $G_{\alpha}^{2}$ values) was relative small than the impact of the rest parameters, we decided to focus on the explanation of the rest parameters only. Therefore, we fixed $\alpha=2$ such that we did not estimate $\alpha$ in our procedure. The significance of the placed cluster center was assessed from 1000-run bootstrap $p$-value of $\Lambda$ at the 0.05 level. The power function at each $\nu$ value was assessed by the rate of significance for $\Lambda$ based on the 1,000 sets.

Location specificity was assessed for all the significant results by the mean Euclidean errors: the greater the distance errors, the less specificity. The mean Euclidean distance between the estimated and the designated point locations is computed as:

$$
M E=\frac{\sum_{k=1}^{1000}\left\|\hat{\mathbf{a}}_{0}-\mathbf{a}_{0}\right\| I_{p_{k}<0.05}}{\sum_{k=1}^{1000} I_{p_{k}<0.05}},
$$

where $p_{k}$ is the bootstrap $p$-value of $k$-runs in the simulation for the selected $\mathbf{a}_{0}$ value.

Figure 3 displays the power function of $\Lambda$ according to a range of $\nu$ values. The type I error probability can be spotted at the point where $\nu=0$. As expected it was close to 0.05 . As $\nu$ increased slightly to 0.1 , the power was less than 0.065. When $\nu$ was between 0.1 and $0.3, \Lambda$ had a weak power to detect the existence of a cluster. As $\nu$ became stronger (between 0.4 to 0.6), the power function increased rapidly from around 0.55 to above 0.95 . It quickly increased to almost 1 when $\nu$ increased to beyond 0.7 .

As expected, location specificity for the point source increases as the cluster strength $\nu$ increases (Figure 4). When the strength was weak with 


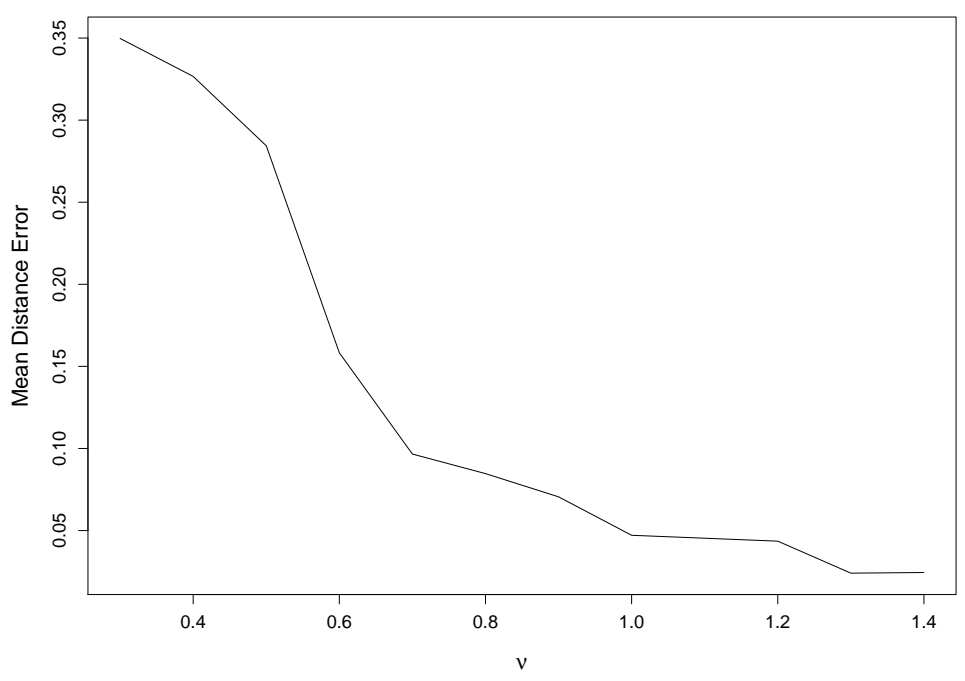

Figure 4: Mean distance error between estimated influence center and the true center. 
small $\nu$ values and few significant results, the estimated points showed spatial randomness, and an average of 0.4 or less shift from the placed point source location was observed. This number was comparable to the expected average of

$$
\int_{0}^{1} \int_{0}^{1} \sqrt{(u-0.5)^{2}+(v-0.5)^{2}} d u d v=0.3826
$$

or the expected distance between a random variable on the unit square to the point location of $(0.5,0.5)$. As $\nu$ increased to 0.7 , the location accuracy was close to 0.1 or one subunit in the unit square. Besides the evaluation of location specificity, we also studied the performance of the estimator of the scale parameter $\gamma$ by looking at $E\left|\hat{\gamma}-\gamma_{0}\right|=E|\hat{\gamma}-0.2|$, which was evaluated by the average of $|\hat{\gamma}-0.2|$ in our simulation. We found that the average of $|\hat{\gamma}-0.2|$ decreased from 0.6490 at $\nu=0.1$ to 0.057 at $\nu=0.7$ indicating that $E|\hat{\gamma}-0.2|$ also decreased as $\nu \rightarrow \infty$.

To briefly summarize, both the power function and the location specificity measures performed satisfactorily according to a range of cluster strengths, and both increased steadily as the strength increased. While the assessment of the power is comparable to the evaluation of other cluster detection power functions, it is worth noting the difference in location specificity between a conventional cluster detection method and the parameterized method. The former uses the center of a geographic unit as the referent. The latter infers the influence center location, not necessarily at the center of a geographic unit. The center of the cluster can be around the intersection of four units if the four are at the core of a cluster. Our parameterization method treats both existence and location of spatial cluster as unknown. Those parameters are continuous in their domains and can be effectively tested and consistently estimated. Our method is able to combine model specification, parameter estimation and hypothesis testing in a unified statistical framework.

\section{Case Study}

As mentioned in the Introduction, a relatively high infant mortality rate in Anhui Province does not fit the profile in the eastern part of China. In this part of the study, we attempt to locate the geographic source of elevated risks by county or county-level city within the province. Anhui has 106 county-level units, which includes 76 rural counties and 30 incorporated city districts. The populations in rural counties and urban districts were 52.4 and 6.6 millions, respectively, with over $99 \%$ being Han ethnic majority. The 
numbers of infant births and deaths in the province from November 1, 1999 to October 31, 2000, were 18,780 and 693,289, respectively. The provincial-level IMR was 27.1 per 1,000; the rates for rural and urban areas were 29 and 9 per 1,000 , respectively. In addition to the rural-urban IMR difference, it was known that infant mortality was high in Dabie Mountain counties (Huaining, Qianshan, Taihu, Susong, Wangjiang and Yuexi), where persistent poverty in the last 100 years caused civil unrest and other social and health problems. Since Dabie mountain counties were the communist basis during the Chinese Civil War, the government has invested heavily in the region to improve the quality of life. However, the IMR in the sparsely populated Dabie Mountain area was still persistently high.

We treated the known rural-urban IMR difference and the high IMR in the Dabie Mountain area with dummy variables. Following the iterative residual approach to cluster detection (Zhang and Lin, 2009a), we progressively fitted a set of models and checked Pearson residuals Moran's I (denoted by $\left.I_{P R}\right)$ for clustering tendency, until we found a model that sufficiently accounted for the existence of spatial clustering. For spatial modeling, the horizontal and vertical axes were based on county centroids of the Universal Transverse Mercator (UTM) coordinates. The UTM coordinate system is the most commonly used among countries in the Northern hemisphere. The original system uses meter as the reference unit, but for the ease of display, we used kilometer. Table 1 summarizes the results from three models that progressively reduced $I_{P R}$ until it was no longer significant.

The first served as a baseline model

$$
M_{1}: \log \left(\lambda_{i}\right)=\log \left(\xi_{i}\right)+\beta_{0}
$$

This is an independence model that corresponds to Model (1) in the method section. This model fitted poorly, with an overall deviance of 3604.1. The $z$-value of $I_{P R}$ was 6.37, suggesting a significant spatial clustering effect on model residuals. Given our knowledge of elevated infant mortality in the Dabie Mountains, these results are not surprising.

Model $M_{2}$ was to account for the rural-urban IMR difference and the high infant mortality in the Dabie Mountain area. However, this model still assumed spatial independence for the rest of counties. Consequently, the model added two additional indicator variables to model $M_{1}$ :

$$
M_{2}: \log \left(\lambda_{i}\right)=\log \left(\xi_{i}\right)+\beta_{0}+\beta_{1} r_{i}+\beta_{2} m_{i}
$$


where $r_{i}=1$ if the $i$-th unit is a rural county and $r_{i}=0$ otherwise; $m_{i}=1$ if county $i$ is within the Dabie Mountain area, and $m_{i}=0$ otherwise. The model (Table 1, column 2) had a significant improvement in the goodness of fit statistic over the previous model, a reduction of more than 1,500 deviance with two additional degrees of freedom. However, the permutation test of Pearson residuals Moran's $I$ still suggested spatial clustering with little change in the standardized $I_{P R}$ and its $p$-value. This led to our next attempt to search for influence centers for other potential clusters by adding the spatial parameterization component to model $M_{2}$ :

$$
M_{3}: \log \left(\lambda_{i}\right)=\log \left(\xi_{i}\right)+\beta_{0}+\beta_{1} r_{i}+\beta_{2} m_{i}+\nu e^{\left(d_{i} / \gamma\right)^{\alpha}} .
$$

Unknown parameters in Model $M_{3}$ can be represented by

$$
\theta=\left(\nu, \beta_{0}, \beta_{1}, \beta_{2}, \gamma, a_{01}, a_{02}, \alpha\right)
$$

where $\left(a_{01}, a_{02}\right)$ are the coordinates of an unknown cluster influence center. Spatial inference can be made from the centroids of clustered counties to the potential influence center $\mathbf{a}_{0}=\left(a_{01}, a_{02}\right)$ by their distance $d_{i}$. The closer a county centroid is to the influence center, the higher the mortality rate.

For any given $\alpha$ in $M_{3}$, we estimated the remaining parameters and computed the values of $G_{\alpha}^{2}$ and $X_{\alpha}^{2}$. We then searched for the best $\alpha$ by minimizing either $G_{\alpha}^{2}$ or $X_{\alpha}^{2}$. The best $\alpha$ value for $G_{\alpha}^{2}$ was 2.12 with $G_{2.12}^{2}=1206.0$, and the best $\alpha$ value for $X_{\alpha}^{2}$ was 2.21 with $X_{2.21}^{2}=1303.3$. Both were very close to 2 . When we used $\alpha=2$, the changes in goodness of fit statistics were ignorable, i.e., $G_{2}^{2}=1206.5$ and $X_{2}^{2}=1303.6$. Coincidentally, this value was exactly the same as the one recommended by two previous studies (Diggle and Elliott, 1997; Katenka, Levina and Michailidis, 2008). For this reason, we chose 2 as the convenient value of $\alpha$. We carried out a test for $H_{0}: \alpha=2$ against $H_{a}: \alpha \neq 2$ about whether the convenient value could be used. The significance of the test could be assessed by the difference between $G_{\alpha}^{2}$ and $G_{2}^{2}$ or the difference between $X_{\alpha}^{2}$ and $X_{2}^{2}$, where the $p$-value could be determined by their asymptotic distributions, which were the $\chi_{1}^{2}$-distribution for both. The result showed that both were not significant accroding to the $p$-values provided by the asymptotic distributions. This implied that our final circular decay model could be based on $\alpha=2$, shown in the third column of Table 1 . Our model was not the same as the model used previously since we treated $\alpha$ as an unknown parameter. Based on a $\chi^{2}$-test in our model, we were able to answer whether the choice of $\alpha=2$ was adequate or not. 
In addition to the exponential decay function, we considered the polynomial decay function in model $M_{3}$, where the last term was modified to $\nu /\left[1+\left(d_{i} / \gamma\right)^{\alpha}\right]$ according to Equation (4). We also searched the best $\alpha$ by minimizing either $G_{\alpha}^{2}$ or $X_{\alpha}^{2}$. The best $\alpha$ value for $G_{\alpha}^{2}$ was 3.55 with $G_{3.55}^{2}=1207.4$ and the best $\alpha$ value for $X_{\alpha}^{2}$ was 3.95 with $X_{3.95}^{2}=1305.1$, which were slightly larger than those in the exponential decay function. Since the differences were small, we concluded that using the exponential decay function and using the polynomial decay function were almost equally better.

To assess if an elliptical decay function fits better than the circular function, we modified the last term in Model $M_{3}$ by using the elliptical distance function at the location with the best circular decay model above. The elliptical distance function is

$$
\begin{aligned}
d_{i}= & {\left[\left(a_{i 1}-a_{01}\right) \cos \omega+\left(a_{i 2}-a_{02}\right) \sin \omega\right]^{2} } \\
& +\left\{c\left[-\left(a_{i 1}-a_{01}\right) \sin \omega+\left(a_{i 2}-a_{02}\right) \cos \omega\right]\right\}^{2},
\end{aligned}
$$

where the angle between the minor axis and horizontal axis was $\omega$ and the ratio between the major radius and minor radius was $c$. Using $\alpha=2$, we had $G_{2}^{2}=2590.4$ and $X_{2}^{2}=2242.5$ in the best elliptical decay model. We then derived $\hat{\omega} \approx 0.0625 \pi, \hat{\gamma} \approx 76.97$, and $\hat{c} \approx 1.37$ by minimizing the $G_{2}^{2}$ value. These results indicated that the angle between the minor axis and horizontal axis was about $11.25^{\circ}$ and the major radius was about $37 \%$ longer than the minor radius. Putting those in the model, we had the elliptical decay function was estimated by

$$
\begin{aligned}
h\left(a_{i 1}, a_{i 2}\right)= & \exp \left\{-\frac{\left(a_{i 1}-474.78\right)^{2}}{6037.76}-\frac{\left(a_{i 1}-474.78\right)\left(a_{i 2}-3624.88\right)}{34004.09}\right. \\
& \left.-\frac{\left(a_{i 2}-3624.88\right)^{2}}{10797.95}\right\} .
\end{aligned}
$$

The results were shown under $M 3$ in Table 1 (Note: In elliptical decay function $\left(M_{3}\right)$, the minor radius is $\gamma$, the major radius is $1.37 \gamma$, and the angle is $\pi / 16)$.

The reduction of the likelihood ratio statistic over $M_{2}$ was 865.1. Based on the bootstrap method described in Section 2.3, we had the $p$-value of the reduction was less than 0.001 , suggesting that the identified cluster influence center in $M_{3}$ can significantly reduce the deviance. The elliptical model fitted significantly better than the circular model with the twice loglikelihood 
Table 1: Results from the known risk and unknown risk parameterization models, where for elliptical decay in $M_{3}$, the minor radius is $\gamma$, the major radius is $1.37 \gamma$, and the angle is $\pi / 16$.

\begin{tabular}{cccccc}
\hline & & & \multicolumn{3}{c}{$M_{3}$} \\
\cline { 4 - 5 } & $M_{1}$ & $M_{2}$ & Circular & Elliptical & Diggle \\
\hline$\nu$ & & & 0.9169 & 0.9697 & 0.9204 \\
$\gamma$ & & & 87.47 & 76.97 & 88.19 \\
$a_{01}$ & & & 474.78 & 474.78 & 476.04 \\
$a_{02}$ & & & 3624.88 & 3624.88 & 3621.10 \\
$\beta_{0}$ & -3.7724 & -3.9830 & -4.6901 & -4.6901 & -4.6901 \\
$\beta_{1}$ & & 0.2251 & 0.8891 & 0.9001 & 0.8877 \\
$\beta_{2}$ & & 0.9488 & 0.4621 & 0.4511 & 0.4635 \\
$G^{2}$ & 3604.1 & 2071.6 & 1206.5 & 1160.1 & 1211.2 \\
$X^{2}$ & 3378.1 & 2022.7 & 1303.3 & 1255.8 & 1308.7 \\
$\Lambda_{G^{2}}$ & & & 865.1 & 901.3 & 870.6 \\
$z_{I_{P R}}$ & 6.366 & 6.361 & 1.501 & 1.148 & 1.97 \\
$p$-value of $I_{P R}$ & $2 \times 10^{-10}$ & $3 \times 10^{-10}$ & 0.1334 & 0.2510 & 0.0488 \\
\hline
\end{tabular}


ratio statistic reduced from 1206.3 to 901.3 , and the major directional effect slightly toward north-north-west. The reduction of the twice loglikelihood ratio statistic was 305.0 with a bootstrap $p$-value for the test of the circular model against the elliptical model less than 0.001 , which was significant. In both cases, the location estimates for $\left(a_{01}\right.$ and $\left.a_{02}\right)$ were 474.78 and 3624.88, respectively. These coordinate values pointed to a cluster center bordering around Fengtai, Panji and Shou administrative units. A strict interpretation would suggest that the elevated risk in the clustered area could be traced to the Tri-Border influence center about 16 kilometers away from the centroid of Fengtai County. Other parameters suggested that the cluster strength was strong with $\nu$ being 0.9169 , and the scalar parameter $\gamma$ being around 87.5 kilometers for the suggested distance influence.

Based on the residuals of model $M_{3}$, the $z$-value for $I_{P R}$ was 1.501 , suggesting that Model $M_{3}$ sufficiently accounted for the clustering effect. As shown in Figure 5, we can assess the expected relative risk at any point within the range of cluster center influence. At the center of the cluster, the relative risk is 2.5. As one moves away from the center, the relative risk declines to 1.4 when the distance to the center is 87.5 and declines to 1.13 when the distance is $2 \times 87.5=175$. As the distance goes to $\infty$, the relative risk declines to 1 . Certainly, the parameterized method can also have a uniform relative risk commonly found in other cluster detection method (e.g., the spatial scan).

There is no single method directly comparable to our approach. To check for model consistency, we used the results from our 'quick' work based on Diggle's method, where $\mathbf{a}_{0}$ is assumed known. In Diggle's method, one only needs to estimate $\beta, \nu$, and $\gamma$. Given that Diggle's method requires a prespecified point source, we partitioned the whole province into 50 by 50 grids, so that each grid cell can be treated as a "known" or potential point source. Based on this partition, we ran $50 \times 50$ Diggle's model according to Model (2), with each prefixed location being the center of each grid cell and with the same distance decay parameter being $\alpha=2$.

After exhausting runs, the best model from Diggle's method (Table 2, last column) was very closed to the result from the proposed parametric method. The distance between the two methods was 3.98 kilometers. All other parameters and goodness of fit statistics were also very close, suggesting that the cluster influence center derived by the parametric method can be used for point source related spatial cluster detection and inference.

We compared the performance of Diggle's method with the performance 


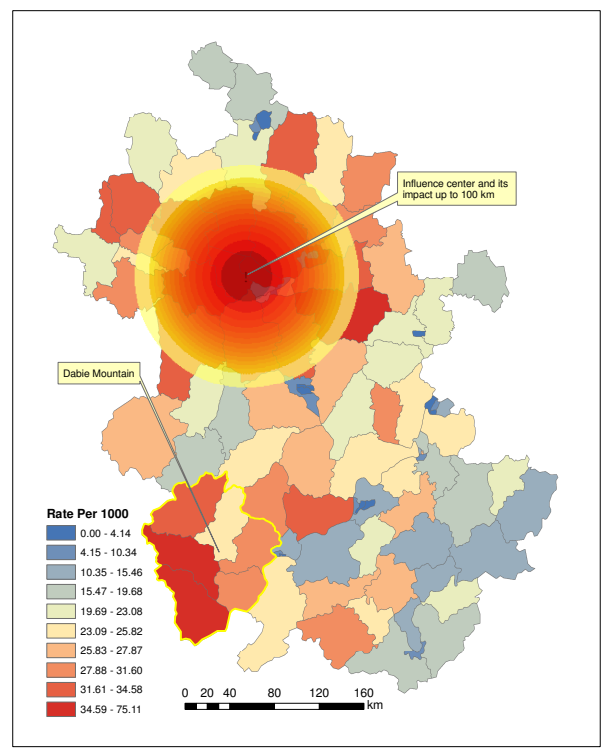

Figure 5: Clusters for Anhui infant mortality.

of our proposed method in the analysis. We found that the parameters $\beta$ and $\nu$ could be easily estimated by the fitting procedures of generalized linear models because they were linear parameters for given $\gamma$ in Diggle's method or for given $\gamma$ and $\mathbf{a}_{0}$ in our proposed method. Therefore, optimization in Diggle's method was easily than optimization in our proposed method as Diggle's method only needed to take care of $\gamma$ in optimization, which is univariate. Since $\gamma$ also disappeared in Diggle's method under the null hypothesis, the asymptotic distribution of the likelihood ratio statistic was still irregular which meant that a bootstrap method was preferred. Since Diggle's method contained a pre-specified point source, we modified this method in our analysis as the location of the point source was unknown. The result from modified Diggle's method was close to the result of our proposed method. Based on this comparison, we concluded that Diggle's method was preferred if the location of the point source was almost known but our proposed method was preferred if the location of the point source was completely unknown. 


\section{Concluding Remarks}

In order to identify cluster strength and its influence center, we have adopted a cluster parameterization approach. Like other spatial cluster parameterization methods, our method can be used for both point and count data. Unlike other methods that needed to specify the point source (Diggle, 1990), our method searches and quantifies unknown cluster influence centers, which adds complexity to the parameterization in terms of estimating multiple parameters. We relied on an iterative specification of the cluster distance decay parameter to estimate the potential cluster location, its strength and influence range, and relied on the bootstrap method to assess parameterized cluster significance. Similar to providing a prior (Wakefield, 2007), GIS can easily index distance and other variables to partition a study area with thousands of known-point sources, and select the model that fits the best. Knowing that this approach is tedious and analytically less appealing, we streamlined computational and statistical process in the current parameterization approach.

The method is able to detect a spatial cluster and to infer a point source that may cause the elevated risk around it. In the analysis, we controlled for a known cluster of the poor Dabie Mountains, and for rural-urban IMR difference, while estimating the model parameters. The method detected a cluster influence center in Fengtai County, an intensive agricultural county with nearby counties engaging in similar intensive agricultural in the west and intensive agriculture and rural-small enterprises in the east. Although we do not know the exact cause of elevated infant mortality, mishandling of agricultural chemicals and malnutrition due to declined breast feeding were likely contributors. Our results assisted the provincial officials to concentrate on the emerging cluster of infant mortality and identify its causes.

There are several limitations of the current study. First, we assumed a distance decay function from a cluster center. Although different distance decay functions had very small effects (Diggle and Elliott, 1997), different functional forms or shapes for spatial pattern estimation should be evaluated in the future (Hall and Kang, 2005). Examples include many geostatistical functional forms, such as directional variograms and correlation functions. Some existing semiparametric and nonparametric estimates provide some insight, such as the visualization method and the slope test-Sizer method (Chauhuri and

Marron, 1990). As the disease mapping method based on existing area units would generate a point estimate for a potential cluster center, a Bayesian es- 
timation procedure could be considered that either complements or provides an alternative to our estimation method. Second, like many previous studies (Morris and Wakefield, 2000), our approach is not an exact method, because we use categorical unit variables to infer the influence of the detected point source and parameters. Even though this method would generally point to the location of a point source, further evaluation for its consistency with small area units are needed in the future. Third, we assume that there is only one spatial cluster in Model (2), but this model can be generalized to include two spatial clusters by using two spatial association terms. However, estimation and numerical algorithms will be complicated by additional spatial association terms and ranking clusters during the fitting process. Finally, our bootstrap method is inefficient, and an analytical asymptotic approach can be pursued in the future (Davies, 2002).

Acknowledgments. The authors appreciate suggestive comments from Associate Editor and Two Reviewers, which significantly improve the quality of the article. The research was funded by US National Science Foundation Grants SES-07-52657 (Zhang) and SES-07-52019 (Lin).

\section{References}

Agresti, A. (1990) Categorical Data Analysis. New York: Wiley.

Besag, J. and Newell, J. (1991) The detection of clusters in rare diseases. J.R. Stat. Soc., A, 154, 143-55.

Boos, D.D. (2003) Introduction to the bootstrap world. Statistical Science, 18, 168-174.

Cai, Y. and William, L. (2007) Child Sex Ratios and their Spatial Variation. In Zhongwei Zhao and Fei Guo (eds.) Transition and Challenge: China's Population at the Beginning of the 21st Century (Oxford University Press), pp. 108-123.

Chaudhuri, P. and Marron, J.S. (1999) SiZer for exploration of structure in curves. JASA, 94, 807-823.

Davies, R. (1997). Hypothesis testing when a nuisance parameter is present only under the alternative. Biometrika, 64, 247-254. 
Davies, R. (2002) Hypothesis testing when a nuisance parameter is present only under the alternative: linear model case. Biometrika, 89, 484-489.

Diggle, P. J. (1990) A point process modelling approach to raised incidence of a rare phenomenon in the vicinity of a prespecified point. Journal of Royal Statistical Society A, 153, 349-362.

Diggle, P. J. and Rowlingson, B. S. (1994) A conditional approach to point process modelling of elevated risk. J. R. Statist. Soc. A, 157, 433-440.

Diggle, P. and Elliott, P. (1997) Regression modelling of disease risk in relation to point sources. Journal of Royal Statistical Society A, 160, 491-505.

Faraway, J. (2005). Extending the Linear Model with R: generalized linear, mixed effects and nonparametric regression models. Chapman \& Hall/CRC, Boca Raton, Florida, United States.

Ferguson, T.S. (1996). A Course in Large Sample Theory. CRC Press, New York.

Gangnon, R.E., and Clayton, M.K. (2003) A hierarchical model for spatially clustered disease ratesStatistics in Medicine, 22, 3213-3228.

Godtliebsen, F., Marron, J.S. and Pizer, S.M. (2002) Significance in scalespace for clustering. Book chapter in Spatial Cluster Modelling, Ed by Lawson, A.B. and Denison, D.G.T., 23-36. Chapman Hall, New York.

Hall, P. and Kang, K. (2005) Unimodal kernel density estimation by data sharpening. Statistica Sinica, 15, 73-98.

Katenka, N., Levina, E. and Michailidis, G. (2008) Local vote decision fusion for target detection in wireless sensor networks. IEEE Transactions on Signal Processing, 56, 329-338.

Kalsall, J. and Diggle, P. (1998) Spatial variation in risk of disease: a nonparametric binary regression approach. Applied Statistics, 4, 3167-3188.

Kulldorff, M. (1997) A spatial scan statistic. Communications in Statistics, Theory and Methods, 26, 1481-1496.

Lawson, A. B. (1993) On the analysis of mortality events associated with a prespecified fixed point. J. R. Statist. Soc. A, 156, 363-377. 
Lawson, A. B. (1995) MCMC methods for putative pollution source problems in environmental epidemiology. Statistics in Medicine, 14:2473-86.

Morris, S.E. and Wakefield J. (2000) Assessment of disease risk in relation to a pre-specified source. Book chapter in Spatial Epidemiology: Methods and Applications Ed by Elliott, P Wakefield, J Best, N and Briggs, D. 153-185. Oxford University Press, Oxford.

Murphy, S. and van der Vaart, A.W. (2000) On profile likelihood. Journal of American Statistical Association, 95, 449-465.

Patefield, W.M. (1977) On the maximized likelihood function. Sankhya B, 39, 92-96.

Peng, R.D. and Bell, M.L. (2010) Spatial misalignment in time series studies of air pollution and health data. Biostatistics. 11, 720-740

Rao, C. R. (1973) Linear Statistical Inference and Its Applications, 2nd Edition. New York: Wiley.

Wakefield, J. (2007) Disease mapping and spatial regression with count data. Biostatistics, 8, 158-183

Wang, F. AND Wall, M. M. (2003) Generalized common spatial factor model. Biostatistics. 4, 569-582.

Wu, Z., Viisainen, K., Li, X and Hemminki, E. (2008) Maternal care in rural China: a case study from Anhui province BMC Health Services Research, 8,55 .

Zhang, T. and Lin, G. (2009a) Cluster detection based on spatial associations and iterated residuals in generalized linear mixed models. Biometrics, 65, 353-360.

Zhang, T. and Lin, G. (2009b) Spatial scan statistics in loglinear model. Computational Statistics and Data Analysis, 53, 2851-2858.

Zhang, T., Zhang, Z., and Ge, L. (2012). Spatial Scan Statistics with Overdispersion. Statistics in Medicine, 31,762-774 